\title{
Cambiando las actitudes hacia la discapacidad: diseño de un programa de sensibilización en Educación Física \\ Changing attitudes towards disability: design of an awareness program in Physical Education \\ Carlos Felipe-Rello, Ignacio Garoz Puerta, Carlos Mª Tejero-González \\ Universidad Autónoma de Madrid (España)
}

\begin{abstract}
Resumen. De acuerdo a los datos de la Organización Mundial de la Salud, un 15\% de la población vive con algún tipo de discapacidad. En España, más de ciento setenta mil estudiantes con necesidades educativas especiales están escolarizados en centros educativos ordinarios. Sin embargo, no abundan los programas de sensibilización hacia la discapacidad que hayan sido adecuadamente validados y publicados en revistas científicas. Este trabajo presenta el diseño y la validación mediante juicio crítico de expertos de un Programa Educativo de Sensibilización hacia las Personas con Discapacidad (ocho sesiones, 50 minutos por sesión), utilizando como herramienta básica la actividad físico-deportiva y valiéndose de cuatro técnicas: información acerca de la discapacidad, contacto directo e indirecto con personas con discapacidad, simulación de diversas discapacidades, y discusión en grupo. Los resultados cuantitativos y cualitativos emitidos por los expertos confirmaron la validación del Programa. Se concluye que el Programa es un recurso práctico, sencillo y eficaz tanto para la formación como para la práctica diaria del profesorado de Educación Física que quiera ser partícipe de una acción docente más transformadora e inclusiva.
\end{abstract}

Palabras clave. Sensibilización, actitudes hacia la discapacidad, educación inclusiva, deporte inclusivo, evaluación de materiales educativos.

\begin{abstract}
According to the World Health Organization, 15\% of the population copes with some type of disability. In Spain, more than one hundred and seventy thousand students with special educational needs are enrolled in ordinary educational centers. However, disability awareness programs that have been adequately validated and published in scientific journals are scarce. This work presents the design and the validation by critical judgment of experts of an Educational Program of Awareness towards People with Disabilities (eight sessions, 50 minutes per session). This Program consists of using sports and physical activity as a tool along with four different techniques: providing information about various disabilities, arranging direct and indirect contact with people with disabilities, incorporating simulations of various disabilities, and finally conducting group discussions. The quantitative and qualitative results issued by experts confirm the validity of the Program. The Program is a practical, simple and effective resource both for training and for the daily practice of Physical Education teachers who want to be part of a more transformative and inclusive teaching approach.
\end{abstract}

Keywords. Awareness, attitudes towards disability, inclusive education, inclusive sport, evaluation of educational materials.

\section{Introducción}

El Informe mundial sobre la discapacidad (Organización Mundial de la Salud, 2011) estima que más de mil millones de personas viven con algún tipo de discapacidad en todo el mundo, lo que supone alrededor del 15\% de la población mundial. Una cifra que se prevé que siga aumentando en el futuro, a pesar de los avances médicos, consecuencia del continuo envejecimiento de la población y al aumento de enfermedades crónicas. Es más, si bien el comienzo del siglo XXI ha supuesto un avance en los derechos humanos de las personas con discapacidad, numerosas organizaciones mundiales se han hecho eco de las desigualdades sociales que todavía imperan en la sociedad por razón de discapacidad, insistiendo por ello en la importancia de promover políticas que favorezcan actitudes de aceptación, respeto e igualdad de las personas con discapacidad. Un claro ejemplo de lo anterior es la Convención sobre los Derechos de las Personas con Discapacidad aprobada por Naciones Unidas en 2006 y ratificada en mayo de 2008, cuyo propósito es «promover, proteger y asegurar el goce pleno y en condiciones de igualdad de todos los derechos humanos y libertades fundamentales por todas las personas con discapacidad, y promover el respeto de su dignidad inherente» (Organización de las Naciones Unidas, 2006, p.4). Por su parte, la Conferencia Mundial sobre Necesidades Educativas Especiales: Acceso y Calidad (Organización de las Naciones Unidas

Fecha recepción: 23-01-19. Fecha de aceptación: 04-07-19 Carlos Felipe Rello cfrello@hotmail.com para la Educación, la Ciencia y la Cultura, 1994) sentó las bases de una educación para todos, asegurando la plena inclusión del alumnado con necesidades educativas especiales en el sistema educativo.

Sin embargo, como señalan algunos autores, el éxito de la inclusión depende en mayor medida de la eliminación de barreras actitudinales que de la implantación de medidas legales, no en vano las actitudes se erigen como una de las barreras más poderosas para la aceptación de las personas con discapacidad (Downs \& Williams, 1994; Lee \& Rodda, 1994; Shannon, Schoen \& Tansey, 2009). Al respecto, la actitud puede ser definida como «una idea cargada de emotividad que predispone una clase de acciones a una clase particular de situaciones sociales» (Triandis, 1974, pp.2-3). De esta definición se pueden extraer tres componentes de las actitudes: un componente cognitivo, un componente afectivo y un componente conductual. La comunidad científica coincide al sostener que las actitudes están en constante cambio, siendo el periodo escolar, durante la infancia y primera adolescencia, cuando los jóvenes desarrollan y consolidan sus actitudes y en donde las intervenciones destinadas a modificar y moldear las actitudes de éstos son más fructíferas (Aguado, Alcedo \& Arias, 2008; Cameron, Rutland, Turner, Holman-Nicolas \& Powell, 2011; Jeon, 2018; Lindsay \& Edwards, 2013).

En la actualidad cada vez son más los alumnos y alumnas con necesidades educativas que se encuentran escolarizados en centros ordinarios. Según los informes que con una periodicidad anual elabora el Consejo Escolar del Estado, durante el curso escolar 2010-2011 el alumnado con necesida- 
des educativas especiales en España suponía un total de 140.215 personas, de las cuales 109.316 estaban integradas en centros ordinarios (Ministerio de Educación, Cultura y Deporte, 2012). Cinco años después, durante el curso escolar 2015-2016, el alumnado con necesidades educativas especiales en España suponía un total de 206.493 personas, de las cuales 171.303 estaban escolarizadas en centros ordinarios (Ministerio de Educación, Cultura y Deporte, 2017), observando un incremento notable, cada vez más significativo, de un colectivo de alumnos y alumnas que demanda necesidades educativas especiales y está escolarizado en entornos inclusivos. Esto demanda a la comunidad educativa una estrategia de actuación basada en los principios de no discriminación, igualdad, normalización y supresión de barreras sociales y arquitectónicas (Ministerio de Sanidad, Servicios Sociales e Igualdad, 2013).

A tenor de estos datos, parece lógico reflexionar sobre la necesidad de incluir programas educativos y de sensibilización dirigidos al alumnado, de manera que estén preparados para recibir y compartir sus aulas con estudiantes con discapacidad. En este sentido, la actividad físico-deportiva se ha estado erigiendo como una herramienta privilegiada para la transmisión de valores y actitudes, normalización, igualdad e inclusión social (Canales, Aravena, CarcamoOyarzun, Lorca \& Martínez-Salazar, 2018; Krahé \& Altwasser, 2006; Pérez-Tejero, Ocete, Ortega-Vila \& Coterón, 2012; Prat \& Soler, 2002; Ríos, 1994; 2006), considerándose a la Educación Física como una de las áreas educativas más proclives al desarrollo de valores y actitudes como contenido curricular, por delante de cualquier otra materia escolar (Velázquez \& Maldonado, 2004). Si bien, es verdad que un estudio reciente apunta que compartir clase de Educación Física con compañeros o compañeras con discapacidad también puede acarrear una disminución de actitudes socialmente deseables, dependiendo de la calidad y planificación del contacto mantenido (Abellán, Sáez-Gallego \& Reina, 2018).

En la actualidad, existen varias organizaciones internacionales y nacionales que sirviéndose de la actividad físicodeportiva trabajan para favorecer la igualdad entre personas con y sin discapacidad y sensibilizar al público en general mediante sendos programas de intervención. A nivel internacional se encuentran el programa Paralympic School Day (International Paralympic Committee, 2014), el Special Olympics Unified Sports (Special Olympics, 2014) o el programa A Touch of Understanding (A Touch Of Understanding, 2014), siendo programas que comparten un objetivo común como es crear conciencia y comprensión hacia las personas con discapacidad a través del deporte inclusivo, propiciando un mayor respeto y aceptación de la diversidad. Similares son los programas que se han puesto en marcha desde instituciones nacionales, como el programa Deporte Inclusivo en la Escuela (Ocete, Pérez-Tejero \& Coterón, 2015), el proyecto Experiencias Paralímpicas (Comité Paralímpico Español, 2014), el programa Tiempos Paralímpicos (El Periódico de Castilla y León, 2016), el programa Incluye-T (Comité Paralímpico Español, Fundación Trinidad Alfonso \& Universidad Miguel Hernández, 2019), el proyecto GaituzSport (Federación Vasca de Deporte Adaptado, 2019) o la Jornada de Sensibilización en Colegios (Fundación Deporte Integra, 2014), sin olvidar el proyecto impulsado por el Instituto de Mayores y Servicios Sociales (IMSERSO), bajo el marco del Programa Europeo Comunitario para el Empleo y la solidaridad PROGRESS (20072013), denominado Sensibilización en centros educativos sobre las personas con discapacidad y mayores como medida de prevención contra la discriminación (De Los Reyes et al., 2008; Vitón et al., 2008).

Dentro de este contexto, son muy pocos los programas correctamente planificados y validados por expertos que estén a disposición de la comunidad educativa para que cualquiera de sus miembros pueda recuperarlos, adaptarlos a su contexto escolar e incluirlos en la programación escolar. Por tal razón se hace pertinente el desarrollo de una herramienta educativa que permita promover la formación de los profesionales de la educación, a fin de poner en marcha programas efectivos de sensibilización destinados a fomentar en su alumnado actitudes receptivas de respeto a los derechos de las personas con discapacidad, tal y como señala el artículo ocho de la Convención sobre los Derechos de las Personas con Discapacidad (Organización de las Naciones Unidas, 2006). El presente trabajo da a conocer el proceso de elaboración y valoración por parte de jueces expertos de un programa educativo de sensibilización destinado al alumnado de Educación Secundaria Obligatoria que, tomando como hilo conductor la actividad físico-deportiva, permita mejorar las actitudes de los estudiantes hacia sus compañeros con discapacidad y hacia la discapacidad en general. De la misma manera, este trabajo pretende ofrecer a los profesionales del campo de la educación una herramienta útil, versátil y funcional que pueda servir de guía para la elaboración de materiales educativos que permitan mejorar las actitudes hacia la discapacidad de la población infantil y adolescente.

\section{Material y método}

El diseño, validación a través del juicio de expertos, y posterior aplicación del programa de sensibilización aquí descrito, denominado Programa Educativo de Sensibilización hacia las Personas con Discapacidad, queda enmarcado en un estudio desarrollado desde la Universidad Autónoma de Madrid, cuyo título corresponde a Actitudes hacia la discapacidad: análisis comparativo del efecto de tres programas de sensibilización en Educación Física. El procedimiento y los resultados de la intervención con el Programa aquí presentado se detallan en Felipe-Rello, Garoz y Tejero-González (2018). No existiendo un modelo único a la hora de planificar y evaluar un programa de intervención y valorando la limitación del número de palabras de este artículo, a continuación se resumirán las dos fases principales: diseño del Programa y validación del mismo por parte de jueces expertos.

\section{Diseño del Programa}

Objetivos del Programa e intenciones educativas de partida

El objetivo principal del Programa Educativo de Sensibilización hacia las Personas con Discapacidad presentado en este trabajo es mejorar las actitudes de la población joven hacia las personas con discapacidad, proporcionándoles 


\begin{tabular}{|c|c|c|c|}
\hline Contenido & Sesiones & Objetivos & Actividades \\
\hline INFORMACIÓN & $\begin{array}{l}\text { Sesión } 2 \\
\text { Diseño universal }\end{array}$ & $\begin{array}{l}\checkmark \text { Conocer estándares básicos de accesibilidad y diseño } \\
\text { universal. } \\
\checkmark \text { Detectar barreras arquitectónicas del entorno. } \\
\checkmark \text { Proponer medidas para hacer el entorno más accesible. }\end{array}$ & $\begin{array}{l}\checkmark \text { Presentación de la sesión. } \\
\checkmark \text { Visionado de documental acerca de la accesibilidad universal. } \\
\checkmark \text { Identificación de barreras arquitectónicas en el Centro. } \\
\checkmark \text { Reflexión y discusión en grupo. }\end{array}$ \\
\hline CONTACTO & $\begin{array}{l}\text { Sesión } 4 \\
\text { Encuentro con un deportista con discapacidad }\end{array}$ & $\begin{array}{l}\checkmark \text { Oír de primera mano cómo se convive con la } \\
\text { discapacidad. } \\
\checkmark \text { Superar ciertos tabúes a la hora de hablar de/con } \\
\text { personas con discapacidad. }\end{array}$ & $\begin{array}{l}\text { Presentación de la sesión. } \\
\checkmark \text { Charla del deportista invitado. } \\
\checkmark \text { Práctica del deporte. } \\
\checkmark \text { Reflexión y discusión en grupo. }\end{array}$ \\
\hline \multirow{2}{*}{ SIMULACIÓN } & $\begin{array}{l}\text { Sesión } 6 \\
\text { Simulación de modalidades deportivas }\end{array}$ & $\begin{array}{l}\checkmark \text { Conocer y practicar diferentes modalidades deportivas } \\
\text { para personas con discapacidad. } \\
\checkmark \text { Experimentar las limitaciones que conllevan ciertas } \\
\text { discapacidades. }\end{array}$ & $\begin{array}{l}\text { Presentación de la sesión. } \\
\checkmark \text { Práctica de diferentes deportes para personas con } \\
\text { discapacidad: goalball, boccia, voleibol sentado. } \\
\checkmark \text { Reflexión y discusión en grupo. }\end{array}$ \\
\hline & $\begin{array}{l}\text { Sesión } 7 \\
\text { Actividad física inclusiva }\end{array}$ & $\begin{array}{l}\checkmark \text { Reflexionar sobre la forma de adaptar diferentes deportes } \\
\text { / juegos a las personas con discapacidad. } \\
\checkmark \text { Potenciar la ayuda y colaboración entre compañeros con } \\
\text { diferentes capacidades. }\end{array}$ & $\begin{array}{l}\checkmark \text { Presentación de la sesión. } \\
\checkmark \text { Reflexión en común y adaptación de tareas a alumnos con } \\
\text { discapacidad. } \\
\checkmark \text { Reflexión y discusión en grupo. }\end{array}$ \\
\hline DISCUSIÓN & $\begin{array}{l}\text { Sesión } 8 \\
\text { Jornada de reflexión }\end{array}$ & $\begin{array}{l}\checkmark \text { Reflexionar y poner en común sentimientos y opiniones } \\
\text { acerca de las personas con discapacidad. } \\
\checkmark \text { Argumentar de manera razonada actitudes y creencias } \\
\text { positivas que favorezcan el respeto y aceptación de las } \\
\text { personas con discapacidad. } \\
\checkmark \text { Valorar conjuntamente el Programa de intervención. }\end{array}$ & $\begin{array}{l}\checkmark \text { Presentación de la sesión. } \\
\checkmark \text { Reflexión y discusión en grupo: valoración del Programa, } \\
\text { percepción final de las personas con discapacidad. }\end{array}$ \\
\hline
\end{tabular}

información sobre la discapacidad, desarrollando la empatía y el sentimiento de valía hacia dicho colectivo, a la vez que tiene la intención de despertar en los jóvenes una actitud crítica y activa frente a las situaciones de discriminación de su entorno próximo.

A la hora de planificar y llevar a la práctica el Programa de intervención, centrado en la modificación de actitudes de la población adolescente hacia las personas con discapacidad, se tuvo en cuenta una serie de intenciones educativas: (1) plantear actividades dirigidas a la modificación de los tres componentes de las actitudes. Acorde con Triandis (1974), las experiencias directas con el objeto de actitud tienden a producir modificaciones en todos los componentes de la actitud, mientras que las experiencias indirectas cambian los componentes perceptivo y conductual; (2) incluir en el diseño, desarrollo y evaluación del programa a profesionales de la educación del ámbito de la discapacidad y a la comunidad educativa: alumnado, profesorado y dirección del centro; (3) establecer acuerdos con entidades, federaciones o instituciones que velen por la inclusión de las personas con

\begin{tabular}{|c|c|}
\hline Contenido & Actividades \\
\hline Información & $\begin{array}{l}\text { Las actividades que conforman este bloque aportan información sobre } \\
\text { las características y peculiaridades de las personas con discapacidad. } \\
\text { Se abordan temas como: conceptos y tipos de discapacidad, entidades } \\
\text { más representativas, legislación de referencia o Juegos Paralímpicos. }\end{array}$ \\
\hline Contacto & $\begin{array}{l}\text { Las actividades en este bloque están centradas en la presentación, ya } \\
\text { sea de forma directa o indirecta, de personas con discapacidad que no } \\
\text { actúan de forma estereotipada. En todos los casos, las actividades están } \\
\text { convenientemente estructuradas y planificadas para evitar el } \\
\text { disconfort, incomodidad o incertidumbre del alumnado. }\end{array}$ \\
\hline Simulación & $\begin{array}{l}\text { Comprende actividades de simulación a modo de juego, como por } \\
\text { ejemplo los deportes paralímpicos, así como la búsqueda conjunta de } \\
\text { adaptaciones para posibilitar la práctica deportiva de personas con } \\
\text { discapacidad. }\end{array}$ \\
\hline Grupo de discusión & $\begin{array}{l}\text { Actividades que ocupan la parte final de cada una de las sesiones y la } \\
\text { última sesión. Son actividades en las cuales se discuten y confrontan } \\
\text { aspectos relacionados con las personas con discapacidad (derechos, } \\
\text { creencias, estereotipos, situaciones discriminatorias, etc.) en un clima } \\
\text { de armonía, cordialidad y respeto. }\end{array}$ \\
\hline
\end{tabular}

discapacidad en el ámbito deportivo; (4) proponer al alumnado experiencias equitativas que satisfagan las necesidades de todos y que puedan ser aplicadas en la vida cotidiana; (5) plantear un programa abierto y flexible, en donde tenga cabida cualquier modificación y adaptación a las distintas realidades; (6) propiciar tiempo dedicado a la reflexión y al diálogo de los participantes, donde el alumnado pueda mostrar una actitud crítica ante la inaccesibilidad del colectivo de personas con discapacidad a los servicios sociales y posibilite la búsqueda de soluciones; y (7) promover especialmente el desarrollo personal y social y no solo el incremento de los niveles de condición y habilidad física, siendo fundamental respetar la individualidad, la participación, la confianza en sí mismos y el esfuerzo.

\section{Selección de contenidos}

La elaboración del Programa comenzó con una revisión sistemática de la bibliografía existente desde 1980 hasta febrero de 2017 en materia de formación y modificación de actitudes hacia las personas con discapacidad. La revisión se llevó a cabo consultando diferentes fuentes: el motor de búsqueda de contenido en Internet Google, y las plataformas de bases de datos SportDiscus, PubMed, PsycINFO y ERIC. Finalmente, se examinaron las referencias bibliográficas de cada uno de los trabajos encontrados. Como descriptores se utilizaron, de forma aislada y combinada, las palabras clave en inglés: attitudes, disability, disabled, handicapped, program, intervention, children, students, physical activity, sport, physical education, y sus equivalentes en español. El patrón de búsqueda atendió a la siguiente combinación: (attitudes)AND (disability OR disabled OR handicapped) AND (program OR programme OR intervention) AND (children OR students OR peers OR pupil) 


Objetivos específicos
$\begin{aligned} & \text { Conocer los aspectos generales de la discapacidad física, } \\ & \text { sensorial e intelectual obteniendo una visión global y } \\ & \text { atendiendo a las características morfológicas, fisiológicas } \\ & \text { y conductuales. }\end{aligned}$
$\begin{aligned} & \text { Conocer los marcos legislativos de referencia en relación a } \\ & \text { las personas con discapacidad. }\end{aligned}$

Incrementar el conocimiento y entendimiento de las personas con discapacidad, así como su relación con la actividad físico-deportiv

personas con discapacid

\section{Identificar personajes públicos que tienen discapacidad y han sobresalido en diferentes ámbitos: científico, deportivo, artístico.}

Conocer herramientas y medios técnicos que facilitan la inclusión de las personas con discapacidad en todos los ámbitos: educativo, deportivo, laboral.

Conocer los Juegos Paralímpicos como el evento deportivo más representativo del deporte para personas con discapacidad.

Con discapacidad. discapacidad.

Conocer y poner en común las creencias y estereotipos ampliamente aceptados hacia las personas con discapacidad.

Identificar y analizar las creencias, estereotipos y prejuicios en relación a las personas con discapacidad, en especial en el ámbito deportivo.

\section{Analizar la discriminación hacia las personas con} discapacidad, y su acceso a la práctica físico-deportiva.

Contactar e interactuar con personas con discapacidad,
acercando al adolescente a la vida de estas personas.
acercando al adolescente a la vida de estas personas.



Simular la discapacidad de la manara más realista posible. Comparar las capacidades propias con las de compañeros y personas con discapacidad, no discriminando en función de las mismas, mostrando voluntad y predisposición para ayudar.

Reflexionar sobre el impacto de estas creencias y prejuicios en las personas con discapacidad.

Identificar situaciones de discriminación concretas (medios de comunicación, educación, acceso laboral...). Potenciar la actitud crítica frente a la inaccesibilidad de las personas con discapacidad.

Dialogar acerca de valores sociales básicos como la igualdad, la dignidad o la justicia.

Conocer y reflexionar sobre la vida de personas co discapacidad que actúan de forma no estereotipada. Practicar diferentes modalidades deportivas junto con personas con discapacidad.

Satisfacer sus necesidades de curiosidad acerca de la vida cotidiana de una persona con discapacidad.

Experimentar algunas de las limitaciones y dificultades que tienen las personas con discapacidad.

Desarrollar alternativas a la práctica deportiva, buscando diversas soluciones a los problemas planteados durante la misma.

Utilizar los medios técnicos y materiales específicos que favorecen la inclusión de las personas con discapacidad e el ámbito deportivo.

Apreciar las capacidades propias y las de compañeros y personas con discapacidad, mostrando una actitud de tolerancia y respeto hacia las mismas.

Aprender a dar ayuda a las personas con discapacidad, fomentándola entre compañeros y hacia las personas con discapacidad.

Papel que juega la legislación en la inclusión de las 3 Identificación de personajes públicos que tienen algún $\quad 1,3$ tipo de discapacidad. Admiración hacia los logros conseguidos por personas con discapacidad.

1,3

Exposición de medios técnicos al servicio de las personas con discapacidad.

1,3

Juegos Paralímpicos: historia, deportes incluidos,
clasificaciones.

Beneficios del deporte para las personas con 3,4 discapacidad.

Creencias, estereotipos, prejuicios: concepto, cómo se 1

Identificación y exposición de creencias comunes en la $\quad 1,3,7,8$

Identificación y exposición de creencias comunes en la $\quad 1,3,7,8$
sociedad.

Argumentación razonada del impacto de creencias y $\quad 1,3,7,8$ $\begin{array}{lc}\begin{array}{l}\text { Arejuicios en las personas con discapacidad. } \\ \text { Análisis de cuál es la realidad con respecto a estos } \\ \text { estereotipos. }\end{array} & 1,3,3,4,8,5,7,8\end{array}$ Elementos del entorno próximo no adaptados a las $2,4,5$ \begin{tabular}{ll} 
personas con discapacidad. & $2,4,5$ \\
\hline Desarrollo de actitudes conscientes frente a situaciones & $2,4,5,8$
\end{tabular} Desarrollo de actitudes conscientes frente a situaciones concretas de discriminación. Propuesta de ante la falta de accesibilidad. $1,2,3,4,8$ Propuesta de cambios que favorezcan la inclusión. $\quad 1,2,4,6,8$ Valores sociales: igualdad, solidaridad, justicia, $\quad 1$ - 8 dignic Conocimiento y reflexión de la vida de las personas $\quad 3,4,5$ con discapacidad. Apreciación de los "problemas/ esfuerzos" a los que $\quad 2,3,4,5,6,8$ tienen que hacer frente las personas con discapacidad. $\quad 2,3,4,5,6,8$ Práctica deportiva conjunta. $\quad 4,5$

Charla-conferencia de una persona con discapacidad. $\quad 4,5$
Intercambios comunicativos, potenciando la claridad,
la educación y el respeto en las intervenciones. Role-playing de situaciones cotidianas (desplazarse por 2 Observación de compañeros que simulan discapacidad $\quad 2,6,7$ y de las reacciones que suscitan. $\quad 2,6$, Conocimiento y práctica simulada de deportes $\quad 6,7$

Búsqueda activa de soluciones ante la necesidad de $\quad 6,7$ daptación de un juego o deporte. Utilización de medios técnicos y materiales específicos discapacidad en el ámbito deportivo.

Comparación de las capacidades propias y las de
aquellos que te rodean.

Respeto y valoración de las capacidades de otros $\quad 2,3,4,5,6,7$ miembros de la comunidad. $\quad 2,3,4,5,6,7$

La asistencia correcta a personas con discapacidad. $\quad 2,4,5,6,7$
Ayuda entre compañeros y hacia las personas con $\quad 2,4,5,6,7$

AND (physical activity OR sport OR physical education). Fueron analizados, entre otros programas, el Paralympic School Day (International Paralympic Committee, 2014), el A Touch Of Understanding (A Touch Of Understanding, 2014) o el Programa PROGRESS (2007-2013) (De los Reyes et al., 2008). Basándose en la revisión realizada se seleccionaron los contenidos propios para la etapa educativa de Educación Secundaria Obligatoria.

Los contenidos del Programa se organizan en cuatro grandes bloques (información, contacto, simulación y discusión) según las diferentes técnicas que se utilizan para modificar las actitudes hacia la discapacidad y que recoge la literatura (Donaldson, 1987; Flórez, Aguado \& Alcedo, 2009; Lindsay \& Edwards, 2013; Verdugo, Arias \& Jenaro, 1994), sin perjuicio de que alguna actividad comprenda varias técnicas. En la Tabla 1 se resume en qué consisten las actividades vinculadas a cada bloque de contenido. A la hora de implementar cada una de las técnicas de cambio de actitudes se tuvieron en consideración una serie de indicaciones y orientaciones que recoge la literatura (Felipe-Rello, Garoz \& Díaz, 2014) para obtener el máximo beneficio de cada una de ellas.

El Programa Educativo de Sensibilización hacia las Personas con Discapacidad se compone de ocho sesiones, cuya duración corresponde a un periodo escolar de 50 minutos, donde de manera progresiva el alumnado se va adentrando en el mundo de la discapacidad. La herramienta educativa que se pone a disposición del profesorado de Educación Física que imparte docencia en la Educación Secundaria Obligatoria consta de una guía didáctica que integra: (1) la propuesta didáctica en sí, donde se detalla la vinculación de la propuesta con el currículo oficial vigente, los objetivos y contenidos de la propuesta, así como la temporalización y los recursos metodológicos y didácticos necesarios; (2) la descripción pormenorizada de las sesiones; (3) una amplia variedad de recursos didácticos, que comprenden presentaciones teóricas en PowerPoint, documentales, spots de cam- 
pañas de sensibilización, bibliografía y webgrafía de referencia, entre otros; y (4) fichas de evaluación para la recogida puntual y veraz de información, por parte del profesorado y alumnado, acerca del devenir de las sesiones y del programa en general. Es necesario señalar que los materiales aportados deben entenderse como una guía de referencia, donde cada centro y/o profesor o profesora tiene que elegir entre todos los contenidos y actividades que se le facilitan y adaptar a las posibilidades del centro y características del alumnado. En la Tabla 2 se exponen las sesiones y objetivos en función de los bloques de contenido. Asimismo, en la Tabla 3 se detallan los objetivos generales y específicos, así como los contenidos y las sesiones donde se ponen en práctica.

\section{Validación de los contenidos del Programa mediante comité de expertos}

Con la finalidad de validar los contenidos del Programa de Sensibilización hacia las Personas con Discapacidad se contó con la colaboración de un grupo de expertos. Para Pérez-Juste (2006), la validación de contenidos por medio de juicio de expertos tiene una doble finalidad: (1) estimar la calidad intrínseca del programa, es decir, determinar si los objetivos y contenidos son adecuados, precisos y coherentes con los demás componentes del programa, y (2) comprobar la viabilidad del programa y adecuación a las características del alumnado y del contexto donde se va a llevar a cabo. Después de estudiar el planteamiento de diferentes autores (Cardoso \& Cerecedo, 2011; Pérez-Juste, 2006), se contempló una serie de indicadores mostrados en la Tabla 4 que deben tomarse en consideración para la valoración de un programa educativo.

Por este motivo, tres jueces expertos participaron en la valoración de los contenidos del Programa. La selección de los expertos atendió a diferentes criterios: (a) contar con expertos de reconocida experiencia en investigación, (b) contar con expertos con amplia experiencia en el ámbito educativo, (c) contar con expertos con amplios conocimientos en metodología de la educación, y (d) contar con expertos con amplia experiencia y conocimiento en discapacidad. De esta manera, el equipo de expertos quedó conformado por un Doctor en Ciencias de la Educación, profesor titular de universidad, Máster en estadística aplicada y partícipe de numerosas publicaciones científicas en revistas de alto impacto; un Doctor en

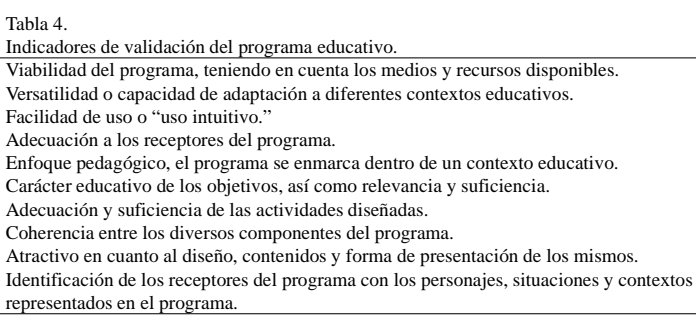

Ciencias de la Actividad Física y el Deporte, profesor contratado de universidad, con más de 20 años de experiencia como profesor de Educación Física y numerosas publicaciones científicas; y una Licenciada en Ciencias de la Actividad Física y el Deporte, Máster en Actividad Físico-Deportiva, Personas con Discapacidad e Integración Social, con una experiencia como profesora de Educación Física superior a 25 años.

Más concretamente, se solicitó al comité de expertos que evaluaran la adecuación o pertinencia del Programa en cada una de las distintas dimensiones en las que estaba dividido el cuestionario de valoración de contenido: (a) propuesta didáctica, (b) sesiones, (c) actividades, (d) fichas de evaluación, (e) recursos didácticos y (f) formato de presentación. Para ello, contaban con una escala de cinco puntos, donde uno correspondía a nada adecuado o nula pertinencia y cinco a totalmente adecuado o máxima pertinencia. También disponían de un espacio en blanco por si veían adecuado exponer alguna información complementaria. Se entiende por pertinencia el «sentido de si el programa da o no, y en el grado adecuado, respuesta a las necesidades de la persona, del grupo humano al que va dirigido» (Pérez-Juste, 2006, p.86). Para los cálculos estadísticos se utilizó el programa informático IBM SPSS Statistics 24. Se procedió con estimaciones de tendencia central y de dispersión.

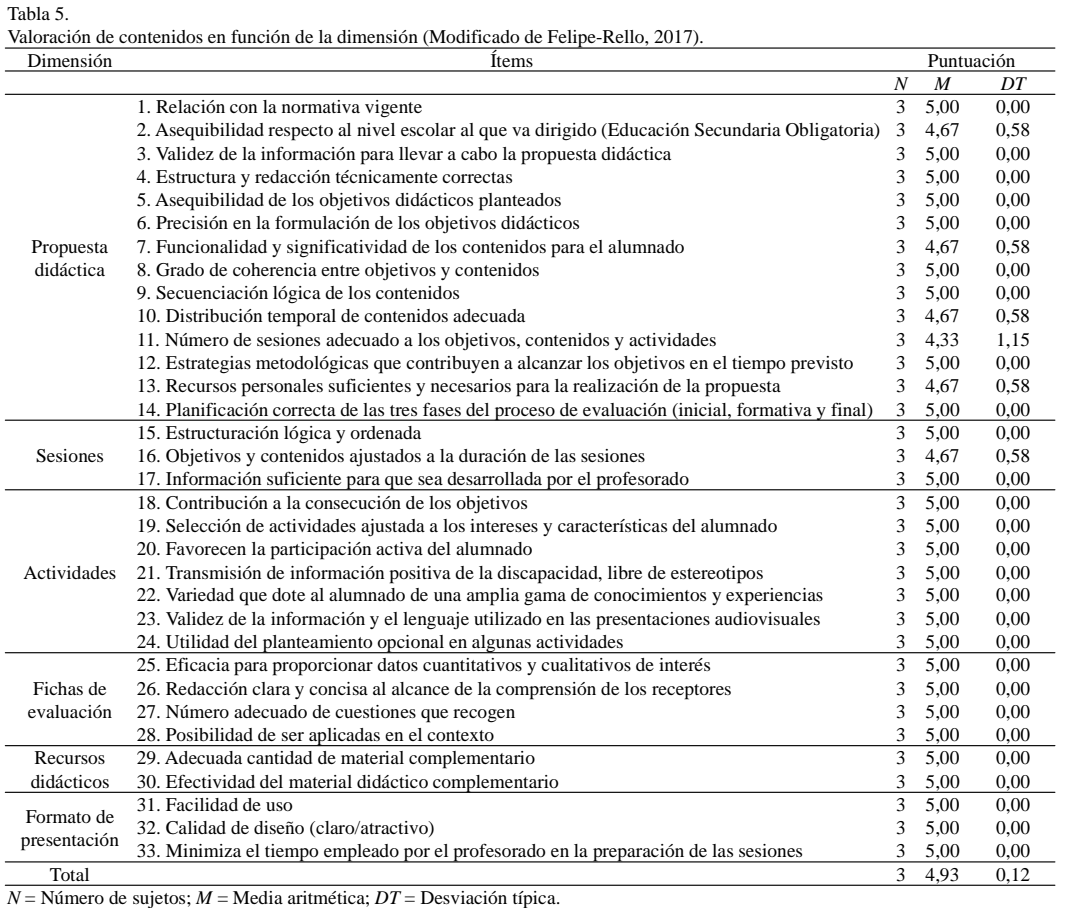

\section{Resultados}

En la Tabla 5 se presenta el cuestionario y se describen los resultados de la valoración de contenidos de cada una de las dimensiones del cuestionario. Los resultados obtenidos muestran que el Programa es válido y adecuado al contexto educativo, obteniendo en su conjunto una puntación media de 4,93 sobre 5 . Cuatro dimensiones obtienen la máxima puntuación (5): actividades, fichas de evaluación, recursos didácticos y formato de presentación. Con una puntuación inferior, pero cercana a la máxima $(4,67)$, se encuentran ítems 
como Asequibilidad respecto al nivel escolar al que va dirigido (Educación Secundaria Obligatoria), Funcionalidad y significatividad de los contenidos para el alumnado, Distribución temporal de contenidos adecuada, Recursos personales suficientes y necesarios para la realización de la propuesta, u Objetivos y contenidos ajustados a la duración de las sesiones. La puntuación inferior, alta en cualquier caso, corresponde al ítem 11: Número de sesiones adecuado a los objetivos, contenidos y activida$\operatorname{des}(4,33)$.

Los datos y consideraciones recogidas en el análisis cualitativo de los cuestionarios de valoración permiten corroborar la utilidad y viabilidad del Programa como herramienta para influir en las actitudes de los jóvenes hacia las personas con discapacidad. Un experto, tal y como se recoge en la siguiente cita, advirtió de la extensión de los contenidos teóricos durante las primeras sesiones y la planificación que requerían dichas sesiones por parte del profesorado:

El Programa es muy ambicioso y requiere del profesor llevarlo muy preparado para ajustarlo al tiempo real de las sesiones de clase, sobre todo en las tres primeras sesiones. La información es extensa y muy interesante.

En referencia a los ítems con puntuaciones inferiores señalar que corresponden gran parte de ellos a la secuenciación temporal de la propuesta didáctica. Los expertos advirtieron de la profundidad de los contenidos y la dificultad para lograrlos en ocho sesiones, siendo más propios de tercero y cuarto curso de la Educación Secundaria Obligatoria:

Considero que es más asequible para alumnos de $3^{\circ} y$ $4^{\circ}$ de la ESO, a partir de 14 años.

Tampoco pasó desapercibido para el grupo de expertos la dificultad para aplicar las sesiones del Programa de forma consecutiva, lo que les llevó a plantear varios interrogantes:

¿Importa que se rompa el ritmo de la unidad didáctica? o ¿No perdería fuerza de impacto si [las sesiones] no son correlativas?

Aunque se instó al profesorado a impartir las sesiones sucesivamente, la distribución de las instalaciones deportivas entre los miembros del Departamento de Educación Física, junto con la disponibilidad de las colaboraciones para las fechas previstas, planteaban serias dudas a la continuidad del Programa. Por ello, se solicitó al profesorado el desarrollo de al menos una sesión del Programa a la semana, pudiendo optar entre las dos horas lectivas que tiene la asignatura de Educación Física semanalmente.

De especial preocupación para los expertos fue la dificultad en la coordinación del Programa. Un programa de estas características exige una exhaustiva planificación de medios materiales, espaciales y personales. La siguiente cita de un experto reconoce tal dificultad:

La secuenciación es perfecta, no debería modificarse, aunque resulte difícil coordinar personas y entidades.

\section{Discusión}

El estudio confirma que el diseño de las herramientas educativas que configuran el Programa Educativo de Sensibilización hacia las Personas con Discapacidad es, a juicio de los expertos participantes en esta investigación, válido para las capacidades y necesidades pedagógicas de la población adolescente escolarizada en centros de enseñanza secundaria. El presente trabajo se erige como uno de los primeros en aportar de manera explícita un índice de objetivos y contenidos secuenciados en el tiempo y evaluados por expertos para lograr un impacto educativo en las actitudes de los escolares hacia las personas con discapacidad. No en vano, como sostienen Castro y Eirín-Nemiña (2018), el área de Educación Física carece de tradición en la investigación centrada en la evaluación de materiales educativos y sus características pedagógicas.

La presente investigación ilustra un enfoque que podría conducir a la incorporación progresiva de actividades de sensibilización hacia colectivos desfavorecidos dentro del currículo de las materias educativas. Tal y como señalan Ocete et al. (2015): «una de las claves para facilitar la concienciación de la situación de las personas con discapacidad en el ámbito educativo es promover actividades en las que las personas sin discapacidad experimenten y aprendan sobre la discapacidad» (p.140). Más concretamente, y dada la relación clara del Programa con la actividad físico-deportiva de las personas con discapacidad, se hace necesario la incorporación de contenidos de Deporte Inclusivo al currículo de Educación Física, tanto en enseñanza primaria como en secundaria, lo cual tiene cabida dentro de la actual Ley Orgánica para la Mejora de la Calidad Educativa (LOMCE) (Ministerio de Educación, Cultura y Deporte, 2013) o, como cabe esperar a tenor de la progresiva sensibilización hacia la discapacidad, en futuras leyes educativas. En este sentido, los deportes inclusivos son una fuente en la transmisión de valores educativos como la sensibilización social, la igualdad de oportunidades o la reflexión crítica, e incluso en mayor medida que los deportes tradicionales (RoblesRodríguez, Abad-Robles, Fuentes-Guerra \& Benito-Peinado, 2017). De la misma manera, el carácter innovador del Deporte Inclusivo hace del propio contenido una actividad novedosa, atractiva y no discriminatoria, al partir todos los alumnos y alumnas del mismo nivel de conocimientos y experiencias previas. No hay que olvidar tampoco la utilidad del Deporte Inclusivo para desarrollar las capacidades psicomotrices del alumnado. Por ejemplo, la realización de determinadas tareas con privación de uno o varios sentidos, o la reducción de la capacidad funcional de una persona, estimulan de manera excepcional otras capacidades y sentidos que no se ven potenciados con la práctica deportiva habitual a la que nos tiene acostumbrado la Educación Física escolar.

De entre las fortalezas de este trabajo, resulta necesario señalar la utilidad y el carácter práctico del Programa por la transferencia o correspondencia entre los planteamientos teóricos y prácticos y la realidad educativa. Un programa de sensibilización continuado en el tiempo, más allá de intervenciones puntuales, puede conducir a una mejora en el ambiente o clima educativo de los centros de enseñanza, a través de una mayor sensibilización hacia la diferencia, ya sea resultante de una discapacidad u otras condiciones de índole religiosa, cultural o de identidad de género. Además, el contenido desarrollado en el Programa permite a los participantes conocer y detectar en su centro educativo o con- 
texto próximo aquellas barreras actitudinales y arquitectónicas que dificultan el acceso a los bienes y servicios para todas las personas en igualdad de condiciones y oportunidades. Asimismo, conviene destacar el carácter flexible del Programa, pudiendo optar por diferentes actividades para lograr un mismo objetivo, la variedad de actividades atendiendo a las cuatro grandes técnicas que la literatura recoge para modificar las actitudes hacia las personas con discapacidad, el bajo coste en términos económicos y, por último, la transferencia o capacidad de extrapolar los aprendizajes a situaciones de la vida real. No en vano, según el Informe mundial sobre la discapacidad(Organización Mundial de la Salud, 2011), casi todas las personas sufrirán en algún momento de su vida algún tipo de discapacidad transitoria o permanente.

Así, como es fácil intuir, un agente clave para desarrollar este tipo de intervenciones es el profesorado, cobrando una vital importancia las políticas de inversión de recursos y esfuerzos en mejorar las actitudes del profesorado hacia la inclusión en el aula y la enseñanza del alumnado con necesidades específicas de apoyo educativo. En este sentido, como afirman distintos autores, la competencia profesional del profesorado y la actitud positiva del mismo han de ser los puntos de partida para una educación inclusiva real, de manera que cualquier actividad de aula destinada a favorecer las actitudes hacia la discapacidad y el autoconcepto de las personas con discapacidad está supeditada al papel del profesorado como agente facilitador de actitudes y comportamientos positivos (Fernández, Jiménez, Navarro \& Sánchez, 2019; Heikinaro-Johansson \& Sherrill, 1994; Salend \& Moe, 1983). Una de las razones que puede justificar las actitudes negativas del personal de los centros educativos hacia los estudiantes con discapacidad es la falta de formación en esteámbito(Kowalski \& Rizzo, 1996; Praisner, 2003). González y Macías (2018), en un estudio conducido con 56 maestros y maestras de Educación Física de educación primaria, subrayan, de manera explícita, las necesidades formativas del profesorado a la hora de trabajar con alumnado con discapacidad. La formación en técnicas de comunicación verbal y no verbal, sistemas de comunicación alternativos o creación de recursos didácticos se encuentran entre las líneas de formación más demandadas. Un profesorado formado, que además disponga de recursos de índole material, económico y humano, contará con las herramientas necesarias para ofrecer una educación de calidad a todos y cada uno de sus alumnos y alumnas, independientemente de sus características, capacidades o limitaciones.

No menos importante es la conveniencia de contar en futuros trabajos de investigación con la ayuda de instituciones y particulares con y sin discapacidad vinculados al objeto de estudio: la actividad físico-deportiva inclusiva. Su implicación en el diseño y desarrollo de los programas harán que sean más válidos y generalizables a la realidad. ¿Quién mejor que las personas con discapacidad para ofrecer una visión equilibrada de la discapacidad? ¿Quiénes pueden estar más interesados que las personas con discapacidad en favorecer valores y actitudes de igualdad, respeto y normalización entre personas con y sin discapacidad? A este respecto, las instituciones educativas deberían ofrecer a sus educandos la oportunidad de compartir experiencias junto con personas con discapacidad. La Teoría del Contacto de Allport (1979) sostiene que el contacto con personas diferentes a uno mismo bajo unas condiciones adecuadas conducirá a un cambio de actitud. Allport especificó cuatro condiciones necesarias para favorecer tal cambio de actitudes: igualdad de estatus, búsqueda de objetivos comunes, interacciones personales significativas y apoyo a nivel institucional y legislativo. Para algunos autores, como McKay (2018), la Teoría del Contacto es la base de muchos programas de intervención, cuyos objetivos son la sensibilización y educación de los escolares hacia la plena inclusión de las personas con discapacidad. Por todo lo apuntado, se insta a los centros educativos e instituciones educativas a buscar, establecer y consolidar posibles nexos de unión con instituciones del ámbito de la discapacidad para desarrollar conjuntamente programas de sensibilización de calidad, de los cuales pudieran obtener beneficios ambas partes.

Como principal limitación o punto débil en el diseño del Programa indicar que no se siguieron de manera fehaciente las recomendaciones de la lista de comprobación PRISMA (PRISMA, 2019) a la hora de conducir la revisión de la bibliografía en busca de programas de intervención basados en la modificación de actitudes hacia las personas con discapacidad, lo que no fue un obstáculo para llevar a cabo una revisión metódica y transparente. Es por ello, que los autores de este trabajo lo proponen como futura línea de investigación en el campo de la modificación de las actitudes hacia la discapacidad. Por su parte, los expertos señalaron la extensión del marco teórico y el escaso tiempo para desarrollar todos los contenidos que albergaban las sesiones. La incorporación de una amplia base de información, referente a la discapacidad y la práctica físico-deportiva de las personas con discapacidad se justifica en la medida en que la información es el paso previo a la puesta en práctica de técnicas de simulación o establecer contacto con personas con discapacidad, como así refleja la literatura (Felipe-Rello et al., 2014). Por otra parte, el objetivo de diseñar sesiones que excedieran en contenidos y actividades al tiempo real de práctica durante una sesión de Educación Física fue poner a disposición del profesorado una amplia cartera de recursos de donde extraer aquellas actividades o propuestas que más se ajustaran a las motivaciones, capacidades e intereses de su alumnado.

Finalmente, es pertinente apuntar la dirección de futuros estudios. En virtud de los resultados obtenidos tras las primeras aplicaciones del Programa de sensibilización aquí presentado (Felipe-Rello et al., 2018), es necesario dar continuidad a este tipo de intervenciones con el fin de mejorar las actitudes hacia las personas con discapacidad. Los resultados mostrados en este y otros estudios confirman no solo los beneficios de llevar a las aulas programas de estas características, sino también la necesidad de promoverlos, como consecuencia de la heterogeneidad del alumnado. Parece oportuno la realización de nuevos estudios que, tomando como referencia trabajos como el aquí presentado, perfeccionen los programas de sensibilización, adoptando nuevas perspectivas que permitan un cambio de actitudes más significativo y duradero. En segundo término, parece adecuada la implementación de programas abordados con un enfoque interdisciplinar, desde el conjunto de las materias que com- 
ponen el currículo, y no solo desde el ámbito de la Educación Física. Finalmente, el Programa de sensibilización está centrado en el ámbito escolar y únicamente sobre el alumnado, desatendiendo otros agentes, como es el entorno familiar, en la modelación de las actitudes de los escolares. En esta línea, numerosos autores reclaman la participación de las familias como un componente vital en la modificación de las actitudes de los más jóvenes hacia la discapacidad y en la inclusión de los niños y niñas con discapacidad en programas de actividad físico-deportiva (Columna, Dillon, Norris, Dolphin \& McCabe, 2017; Favazza \& Odom, 1997; Navas, Torregrosa \& Mula, 2004; Simpson, 1980).

\section{Conclusiones}

En conclusión, el Programa de Sensibilización hacia las Personas con Discapacidad presentado en este trabajo constituye una herramienta válida, sencilla, práctica y versátil que pretende complementar la formación del profesorado en el ámbito de la discapacidad y, a la vez, beneficiar al alumnado en general, fortaleciendo la aceptación e inclusión en las aulas de compañeros y compañeras con capacidades diferentes. Y, con ello, ayudar a construir entre todos una escuela para todos y con todos, libre de barreras actitudinales y falsas creencias.

\section{Agradecimientos}

Especial agradecimiento al grupo de expertos que participó en la valoración de los materiales y contenidos del Programa.

\section{Referencias}

A Touch Of Understanding. (2014). A Touch Of Understanding. Recuperado de http://www.touchofunderstanding.org/

Abellán, J., Sáez-Gallego, N. M., \& Reina, R. (2018). Evaluación de las actitudes hacia la discapacidad en Educación Física: efecto diferencial del sexo, contacto previo y la percepción de habilidad y competencia. Cuadernos de Psicología del Deporte, 18(1), 133-140. doi: 10.5232/ricyde2018.05304

Aguado, A. L., Alcedo, M. A., \& Arias, B. (2008). Cambio de actitudes hacia la discapacidad con escolares de Primaria. Psicothema, 20(4), 697-704.

Allport, G. W. (1979). The nature of prejudice. Cambridge, Massachusetts: Perseus Books.

Cameron, L., Rutland,A., Turner, R., Holman-Nicolas, R., \& Powell, C. (2011). Changing attitudes with a little imagination: imagined contact effects on young children's intergroup bias. Anales de Psicología, 27(3), 708-717.

Canales, P., Aravena, O., Carcamo-Oyarzun, J., Lorca, J., \& Martínez-Salazar, C. (2018). Prácticas pedagógicas que favorecen u obstaculizan la inclusión educativa en el aula de educación física desde la perspectiva del alumnado y profesorado. Retos. Nuevas tendencias en Educación Física, Deportes y Recreación, 34, 212-217.

Cardoso, E., \& Cerecedo, M. (2011). Propuesta de indicadores para evaluar la calidad de un programa de posgrado en Educación. Revista Electrónica de Investigación Educativa, 13(2), 68-82.

Castro, M. M., \& Eirín-Nemiña, R. (2018). Evaluación de materiales de educación física para la atención a la diversidad. Análisis de una propuesta. Retos. Nuevas tendencias en Educación Física, Deportes y Recreación, 34, 356-362.

Columna, L., Dillon, S. R., Norris, M. L., Dolphin, M., \& McCabe, L. (2017). Parents' perceptions of physical activity experiences for their families and children with visual impairments. British Journal of Visual Impairment, 35(2), 88-102. doi: 10.1177/ 0264619617691081

Comité Paralímpico Español. (2014). Proyectos-Experiencias Paralímpicas. Recuperado de http://paralimpicos.es/ publicacion/11SC_proyectos/301SS_Experiencias.asp

Comité Paralímpico Español, Fundación Trinidad Alfonso \& Universidad Miguel Hernández. (2019). Incluye-T. Educación Física y Deporte Inclusivo. Recuperado de https:// incluyeteblog.wordpress.com/

De Los Reyes, J. L., Vitón, M. J., Calero, S., Sellés, M. R., Villagra, H. A., \& Luna, L. (2008). Sensibilización en centros educativos sobre las personas con discapacidad y mayores como medida de prevención contra la discriminación. Guía de recursos didácticos en Educación Primaria. Madrid: Instituto de Mayores y Servicios Sociales (IMSERSO).

Donaldson, J. (1987). Cambio de actitudes hacia las personas deficientes. Siglo Cero, 112, 30-38 (Versión en castellano del original de 1980: Changing attitudes toward handicapped persons: a review and analysis of research. Exceptional Children, 46, 504-514).

Downs, P., \& Williams, T. (1994). Student attitudes toward integration of people with disabilities in activity settings: a European comparison. Adapted Physical Activity Quarterly, 11(1), 32-43. doi: 10.1123/apaq.11.1.32

El Periódico de Castilla y León. (2016). La cuarta edición de Tiempos Paralímpicos va dirigida a centros dedicados al alumnado con discapacidad. Recuperado de http:// elperiodicodecastillayleon.com/noticia/2016-03-18-la-cuartaedicion-tiempos-paralimpicos-va-dirigida-centros-dedicadosal-alumnado-discapacidad-1592

Favazza, P. C., \& Odom, S. L. (1997). Promoting positive attitudes of kindergarten-age children toward people with disabilities. Exceptional Children, 63(3), 405-418. doi: 10.1177/ 001440299706300308

Federación Vasca de Deporte Adaptado. (2019). Gaituzsport. Recuperado de http://www.gaituzsport.eus

Felipe-Rello, C. (2017). Actitudes hacia la discapacidad: análisis comparativo del efecto de tres programas de sensibilización en Educación Física (Tesis doctoral no publicada). Facultad de Formación de Profesorado y Educación, Universidad Autónoma de Madrid.

Felipe-Rello, C., Garoz, I., \& Díaz, M. (2014). Determinantes de las técnicas de cambio de actitudes hacia la discapacidad en programas educativos: una revisión internacional. Siglo Cero, 45(3), 28-46.

Felipe-Rello, C., Garoz, I., \& Tejero-González, C. M. (2018). Análisis comparativo del efecto de tres programas de sensibilización hacia la discapacidad en Educación Física. Retos. Nuevas tendencias en Educación Física, Deportes y Recreación, 34, 258-262.

Fernández, J. M., Jiménez, F., Navarro, V., \& Sánchez, C. R. (2019). Cambios en el autoconcepto del alumnado con y sin discapacidad motriz a partir de una intervención docente inclusiva en educación física. Retos. Nuevas tendencias en Educación Física, Deportes y Recreación, 36, 138-145.

Flórez, M. A., Aguado, A. L., \& Alcedo, M. A. (2009). Revisión y análisis de los programas de cambio de actitudes hacia las personas con discapacidad. Anuario de Psicología Clínica y de la Salud, 5, 85-98.

Fundación Deporte Integra. (2014). Jornada de Sensibilización en 
Colegios. Recuperado de http://www.deporteintegra.eu/modules/content/index.php?id=11\&page= eventos-de-colegios

González, I., \& Macías, D. (2018). La formación permanente como herramienta para mejorar la intervención del maestro de educación física con alumnado con discapacidad. Retos. Nuevas tendencias en Educación Física, Deportes y Recreación, 33, 118122.

Heikinaro-Johansson, P., \& Sherrill, C. (1994). Integrating children with special needs in physical education: a school district assessment model from Finland. Adapted Physical Activity Quarterly, 11(1), 44-56. doi: 10.1123/apaq.11.1.44

International Paralympic Committee. (2014). Paralympic School Day. Recuperado de http://www.paralympic.org/the-ipc/ paralympic-school-day

Jeon, M. (2018). Meta-analysis of disability simulation research for elementary students in Korea. International Journal of Special Education, 33(1), 140-151.

Kowalski, E. M., \& Rizzo, T. L. (1996). Factors influencing preservice student attitudes toward individuals with disabilities. Adapted Physical Activity Quarterly, 13(2), 180-196. doi: 10.1123/apaq.13.2.180

Krahé, B., \& Altwasser, C. (2006). Changing negative attitudes towards persons with physical disabilities: an experimental intervention. Journal of Community \& Applied Social Psychology, 16(1), 59-69. doi: 10.1002/casp.849

Lee, T., \& Rodda, M. (1994). Modification of attitudes toward people with disabilities. Canadian Journal of Rehabilitation, 7(4), 229-238.

Lindsay, S., \& Edwards, A. (2013). A systematic review of disability awareness interventions for children and youth. Disability \& Rehabilitation, 35(8), 623-646. doi: 10.3109/ 09638288.2012.702850

McKay, C. (2018). The value of contact: unpacking Allport's Contact Theory to support inclusive education. Palaestra, 32(1), 21-25.

Ministerio de Educación, Cultura y Deporte. (2012). Informe 2012 sobre el estado del sistema educativo. Curso 2010 2011. Recuperado de https://www.mecd.gob.es/dam/jcr:3a7a949f-6c3f4cf3-907d-f8320c4b8b92/i2012cee-pdf.pdf

Ministerio de Educación, Cultura y Deporte. (2013). Ley Orgánica 8/2013 para la mejora de la calidad educativa. Boletín Oficial del Estado de 10 de diciembre.

Ministerio de Educación, Cultura y Deporte. (2017). Informe 2017 sobre el estado del sistema educativo. Curso 2015 2016. Recuperado de http://ntic.educacion.es/cee/informe2017/ i17cee_informe.pdf

Ministerio de Sanidad, Servicios Sociales e Igualdad. (2013). Real Decreto Legislativo 1/2013, de 29 de noviembre, por el que se aprueba el Texto Refundido de la Ley General de derechos de las personas con discapacidad y de su inclusión social. Boletín Oficial del Estado de 29 de noviembre.

Navas, L., Torregrosa, G., \& Mula. A. (2004). Algunas variables predictoras de las actitudes del alumnado ante la integración escolar. Revista de Psicología Social, 19(2), 159-171.

Ocete, C., Pérez-Tejero, J., \& Coterón, J. (2015). Propuesta de un programa de intervención educativa para facilitar la inclusión de alumnos con discapacidad en educación física. Retos. Nuevas tendencias en Educación Física, Deportes y Recreación, $27,140-145$.

Organización de las Naciones Unidas. (2006). Convención sobre los Derechos de las Personas con Discapacidad y Protocolo Facultativo. Recuperado de http://www.un.org/disabilities/ documents/convention/convoptprot-s.pdf

Organización de las Naciones Unidas para la Educación, la Ciencia y la Cultura. (1994). Conferencia Mundial sobre Necesidades
Educativas Especiales: Acceso y Calidad. Recuperado de http:/ /unesdoc.unesco.org/images/0011/ 001107/110753so.pdf

Organización Mundial de la Salud. (2011). Informe mundial sobre la discapacidad. Recuperado de http://www1.paho.org/arg/ images/Gallery/Informe_spa.pdf

Pérez-Juste, R. (2006). Evaluación de programas educativos. Madrid: La Muralla.

Pérez-Tejero, J., Ocete, C., Ortega-Vila, G., \& Coterón, J. (2012). Diseño y aplicación de un programa de intervención de práctica deportiva inclusiva y su efecto sobre la actitud hacia la discapacidad: el Campus Inclusivo de Baloncesto. Revista Internacional de Ciencias del Deporte, 29(8), 258-271. doi: 10.5232/ricyde2012.02905

Praisner, C. L. (2003). Attitudes of elementary school principals toward the inclusion of students with disabilities. Exceptional Children, 69(2), 135-145. doi: 10.1177/001440290306900201

Prat, M., \& Soler, S. (2002). Las posibilidades del juego, la actividad física y el deporte para la mejora de la convivencia. Revista Electrónica Interuniversitaria de Formación del Profesorado, 5(2), 1-5. Recuperado de http://www.aufop.com/aufop/ uploaded_files/articulos/1227715137.pdf

PRISMA. (2019). Declaración Preferred Reporting Items for Systematic Reviews and Meta-Analyses (PRISMA). Recuperado de http://www.prisma-statement.org/

Ríos, M. (1994). Los juegos sensibilizadores: una herramienta de integración social. Apunts, 38, 93-98.

Ríos, M. (2006). Actividad física adaptada. El juego y los alumnos con discapacidad. La integración en los juegos. Juegos específicos. Juegos motrices sensibilizadores ( $5^{\mathrm{a}}$ ed.). Barcelona: Paidotribo.

Robles-Rodríguez, J., Abad-Robles, M. T., Fuentes-Guerra, J. G., \& Benito-Peinado, P. (2017). Los deportes adaptados como contribución a la educación en valores y a la mejora de las habilidades motrices: la opinión de los alumnos de Bachillerato. Retos. Nuevas tendencias en Educación Física, Deportes y Recreación, 31, 140-144.

Salend, S. J., \& Moe, L. (1983). Modifying nonhandicapped students' attitudes toward their handicapped peers through children's literature. Journal for Special Educators, 19(3), 2228.

Shannon, C. D., Schoen, B., \& Tansey, T. N. (2009). The effect of contact, context, and social power on undergraduate attitudes toward persons with disabilities. Journal of Rehabilitation, 75(4), 11-18.

Simpson, R. L. (1980). Modifying the attitudes of regular class students toward the handicapped. Focus on Exceptional Children, 13(3), 1-11.

Special Olympics. (2014). Special Olympics Unified Sports. Recuperado de http://www.specialolympics.org/unified-sports.aspx

Triandis, H. C. (1974). Actitudes y cambios de actitudes. Barcelona: Toray.

Velázquez, R., \& Maldonado,A. (2004). Las actitudes y su evaluación en educación física. En J. L. Hernández y R. Velázquez (Coords.), La evaluación en educación física. Investigación y práctica en el ámbito escolar (pp. 169-203). Barcelona: GRAO.

Verdugo, M. A., Arias, B., \& Jenaro, C. (1994). Actitudes hacia las personas con minusvalía. Madrid: Ministerio de Asuntos Sociales, Instituto Nacional de Servicios Sociales.

Vitón, M. J., De Los Reyes, J. L., Sellés, M. R., Calero, S., Villagra, H. A., \& Luna, L. (2008). Sensibilización en centros educativos sobre las personas con discapacidad y mayores como medida de prevención contra la discriminación. Guía de recursos didácticos en Educación Infantil. Madrid: Instituto de Mayores y Servicios Sociales (IMSERSO). 